\title{
Expansions for nearly Gaussian distributions
}

\author{
S. Blinnikov ${ }^{1,2}$ and R. Moessner ${ }^{2}$ \\ 1 Institute for Theoretical and Experimental Physics, 117259, Moscow, Russia \\ Sternberg Astronomical Institute, 119899 Moscow, Russia \\ 2 Max-Planck-Institut für Astrophysik, D-85740 Garching, Germany
}

Received July 8; accepted November 10, 1997

\begin{abstract}
Various types of expansions in series of Chebyshev-Hermite polynomials currently used in astrophysics for weakly non-normal distributions are compared, namely the Gram-Charlier, Gauss-Hermite and Edgeworth expansions. It is shown that the Gram-Charlier series is most suspect because of its poor convergence properties. The Gauss-Hermite expansion is better but it has no intrinsic measure of accuracy. The best results are achieved with the asymptotic Edgeworth expansion. We draw attention to the form of this expansion found by Petrov for arbitrary order of the asymptotic parameter and present a simple algorithm realizing Petrov's prescription for the Edgeworth expansion. The results are illustrated by examples similar to the problems arising when fitting spectral line profiles of galaxies, supernovae, or other stars, and for the case of approximating the probability distribution of peculiar velocities in the cosmic string model of structure formation.
\end{abstract}

Key words: methods: statistical; cosmic strings; line: profiles

\section{Introduction}

The normal, or Gaussian, distribution plays a prominent role in statistical problems in various fields of astrophysics and general physics. This is quite natural, since the sums of random variables tend to a normal distribution when the quite general conditions of the central limit theorem are satisfied. In many applications, to extract useful information on the underlying physical processes, it is more interesting to measure the deviations of a probability density function (hereafter PDF) from the normal distribution than to prove that it is close to the Gaussian one. This has been done for example in the work on peculiar velocities and cosmic microwave background anisotropies in various cosmological models (Scherrer \&

Send offprint requests to: S. Blinnikov
Bertschinger 1991; Kofman et al. 1994; Moessner et al. 1994; Juszkiewicz et al. 1995; Bernardeau \& Kofman 1995; Amendola 1994; Colombi 1994; Moessner 1995; Ferreira et al. 1997; Gaztañaga et al. 1997), in analyzing the velocity distributions and fine structure in elliptical galaxies (Rix \& White 1992; van der Marel \& Franx 1993; Gerhard 1993; Heyl et al. 1994), and in studies of large Reynolds number turbulence (Tabeling et al. 1996). In this paper we present a unified approach to the formalism used in those applications, illustrating it by examples of similar problems arising in cosmology and in the theory of supernova line spectra.

The first investigation of slightly non-Gaussian distributions was undertaken by Chebyshev in the middle of the 19th century. He studied in detail a family of orthogonal polynomials which form a natural basis for the expansions of these distributions. A few years later the same polynomials were also investigated by Hermite and they are now called Chebyshev-Hermite or simply Hermite polynomials (their definition was first given by Laplace, see e.g. Encyclopaedia of Mathematics 1988).

There are several forms of expansions using Hermite polynomials, namely the Gram-Charlier, Gauss-Hermite and Edgeworth expansions. We introduce the notation in Sect. 2 and use the simple example of the $\chi^{2}$ distribution for various degrees of freedom to illustrate the properties of Gram-Charlier expansions in Sect. 3. In subsequent sections the $\chi^{2}$ distribution is used for testing the other two expansions. We show in Sect. 4 that the Gram-Charlier series is just a Fourier expansion which diverges in many situations of practical interest, whereas the Gauss-Hermite series has much better convergence properties. However, we point out that another series, the so called Edgeworth expansion, is more useful in many applications even if it is divergent, since, first, it is directly connected to the moments and cumulants of a PDF (the property which is lost in the Gauss-Hermite series) and, second, it is a true asymptotic expansion, so that the error of the approximation is controlled. 
Some applications (e.g. Bernardeau 1992, 1994; Moessner 1995) involve cumulants of higher order. These require the use of a correspondingly higher order Edgeworth series, but only the first few terms of the series are given in standard references (Cramér 1957; Abramowitz \& Stegun 1972; Juszkiewicz et al. 1995; Bernardeau \& Kofman 1995). In Sect. 5 we popularize a derivation of the Edgeworth expansion due to Petrov $(1962,1972,1987)$ for an arbitrary order of the asymptotic parameter, and we present a slightly simpler and more straightforward way of obtaining his result. The formula found by Petrov (see Sect. 5 below) requires a summation over indices with non-trivial combinatorics which hindered its direct application. We find a simple algorithm realizing Petrov's prescription for any order of the Edgeworth expansion; this algorithm is easily coded, e.g. with standard Fortran, eliminating the need for symbolic packages.

We find that the Edgeworth-Petrov expansion is indeed very efficient and reliable. In Sect. 6 we apply the formalism to the problem of peculiar velocities resulting from cosmic strings studied by Moessner (1995) and we show how this technique allows one to reliably extract deviations from Gaussianity, even when they are tiny.

\section{Background and notation}

Let $F(x)$ be the cumulative probability distribution of a random variable $X$. Then the mean value for the random variable $g(X)$ is the expectation value

$\mathrm{E} g(X) \equiv\langle g(X)\rangle \equiv \int_{-\infty}^{\infty} g(x) \mathrm{d} F(x)$.

The PDF is $p(x)=\mathrm{d} F(x) / \mathrm{d} x$. The distribution $F(x)$ is not necessarily smooth, so it can happen that $p(x)$ is nonexistent at certain points. Nevertheless, the mean $\mathrm{E} g(X)$ is defined as long as the integral in (1) exists. Following the definition (1), the $k$-th order moment of $X$ is

$\alpha_{k} \equiv \mathrm{E} X^{k}=\int_{-\infty}^{\infty} x^{k} \mathrm{~d} F(x)$

Thus, the mean of $X$ is $m \equiv \alpha_{1}=\mathrm{E} X$ and its dispersion is

$\sigma^{2} \equiv \mathrm{E}(X-\mathrm{E} X)^{2}=\int_{-\infty}^{\infty}(x-m)^{2} \mathrm{~d} F(x)$.

We denote the cumulative normal distribution by

$P(x) \equiv \int_{-\infty}^{x} Z(t) \mathrm{d} t=\frac{1}{\sqrt{2 \pi}} \int_{-\infty}^{x} \exp \left(-t^{2} / 2\right) \mathrm{d} t$,

so its PDF is the Gaussian function

$Z(x)=\frac{\exp \left(-x^{2} / 2\right)}{\sqrt{2 \pi}}$.

We also need to recall some definitions for sets of orthogonal polynomials. Any two polynomials $P_{n}(x)$ and $P_{m}(x)$ of degrees $n \neq m$ are orthogonal on the real axis with respect to the weight function $w(x)$ if

$\int_{-\infty}^{\infty} w(x) P_{n}(x) P_{m}(x)=0$.

We follow the notation of Abramowitz \& Stegun (1972) where possible, so $H_{n}(x)$ denotes the polynomial with weight function $w(x)=\exp \left(-x^{2} / 2\right) \propto Z(x)$. According to Rodrigues' formula,

$H e_{n}(x)=(-1)^{n} \mathrm{e}^{x^{2} / 2} \frac{\mathrm{d}^{n}}{\mathrm{~d} x^{n}} \mathrm{e}^{-x^{2} / 2}$

We will refer to $\mathrm{He}_{n}(x)$ as Chebyshev-Hermite polynomials following Kendall (1952). The ones with the weight $w(x)=\exp \left(-x^{2}\right) \propto Z^{2}(x)$,

$H_{n}(x)=(-1)^{n} \mathrm{e}^{x^{2}} \frac{\mathrm{d}^{n}}{\mathrm{~d} x^{n}} \mathrm{e}^{-x^{2}}$,

will be called Hermite polynomials.

The expansions in Chebyshev-Hermite and Hermite polynomials are used in many applications in astrophysics. For a PDF $p(x)$ which is nearly Gaussian, it seems natural to use the expansion

$p(x) \sim \sum_{n=0}^{\infty} c_{n} \frac{\mathrm{d}^{n} Z(x)}{\mathrm{d} x^{n}}$,

where the coefficients $c_{n}$ measure the deviations of $p(x)$ from $Z(x)$. From the definitions (5) and (7) it follows that $\frac{\mathrm{d}^{n} Z(x)}{\mathrm{d} x^{n}}=(-1)^{n} H e_{n}(x) Z(x)$,

so that

$p(x) \sim \sum_{n=0}^{\infty}(-1)^{n} c_{n} H e_{n}(x) Z(x)$,

with

$c_{n}=\frac{(-1)^{n}}{n !} \int_{-\infty}^{\infty} p(t) H e_{n}(t) \mathrm{d} t$.

This is the well-known Gram-Charlier series (of type A, see e.g. Cramér 1957; Kendall 1952 and references therein to the original work). Since $H e_{n}(x)$ is a polynomial, we see that the coefficient $c_{n}$ in (12) is a linear combination of the moments $\alpha_{k}$ of the random variable $X$ with $\mathrm{PDF}$ $p(x)$. The combination is easily found by using the explicit expression for $\mathrm{He}_{n}(x)$, which we derive in Appendix B:, namely

$H e_{n}(x)=n ! \sum_{k=0}^{[n / 2]} \frac{(-1)^{k} x^{n-2 k}}{k !(n-2 k) ! 2^{k}}$.

The Gram-Charlier series was used in cosmological applications in the paper by Scherrer \& Bertschinger (1991), but note that it is incorrectly called Edgeworth expansion there. The Gram-Charlier series is merely a kind of Fourier expansion in the set of polynomials $H_{n}(x)$. This expansion has poor convergence properties (Cramér 1957). Very often, for realistic cases, it diverges violently. We consider such an example in the next section. The Edgeworth series, discussed in detail in Sect. 5, is a true asymptotic expansion of the PDF, which allows one to control its accuracy. 


\section{An example based on the $\chi^{2}$ distribution}

A good example for illustrating the fast divergence of the Gram-Charlier series is given by its application to the $\chi_{\nu}^{2}$ distribution with $\nu$ degrees of freedom, since the moments of this distribution are known analytically and its PDF tends to the Gaussian one for large $\nu$. If $X_{1}, X_{2}, \ldots, X_{\nu}$, are independent, normally distributed random variables with zero expectation and unit dispersion, then the variable $\chi^{2}=\chi_{\nu}^{2} \equiv \sum_{i=1}^{\nu} X_{i}^{2}$ has the PDF

$\rho\left(\chi^{2}\right)=\frac{\left(\chi^{2}\right)^{\nu / 2-1} \exp \left(-\chi^{2} / 2\right)}{2^{\nu / 2} \Gamma(\nu / 2)}, \quad \chi^{2}>0$.

The expectation value of $\chi^{2}$ is $\nu$ and its dispersion is $2 \nu$. If $x=\left(\chi^{2}-\nu\right) / \sqrt{2 \nu}$, then $x$ has zero expectation and unit dispersion and its $\operatorname{PDF} p(x)$ asymptotically tends to the Gaussian distribution $Z(x)$. Transforming $\rho\left(\chi^{2}\right)$ from (14) to $p(x)$, we obtain for $x>-\sqrt{\nu / 2}$ :

$p(x)=\sqrt{2 \nu} \frac{(\sqrt{2 \nu} x+\nu)^{\nu / 2-1} \exp -(\sqrt{2 \nu} x+\nu) / 2}{2^{\nu / 2} \Gamma(\nu / 2)}$.

The $\chi^{2}$ distribution was employed by Matsubara \& Yokoyama (1996) for a representation of the cosmological density field (see also Luo 1995 for an application of the $\chi^{2}$ distribution in the context of cosmic microwave background temperature anisotropies). It can also serve as a crude model for approximating the profiles of spectral lines in moving media. If the lines are nearly Gaussian in the matter at rest, then the motion with a high velocity gradient, like that in supernova envelopes, leads to distortions which can be approximated by the $\chi_{\nu}^{2} \operatorname{PDF}$ (with $\nu=2$ for highly non-Gaussian profiles, see e.g. Blinnikov 1996, and references therein).

The comparison of the Gram-Charlier approximations of $p(x)$ with the exact results is presented in Figs. 1 and 2 for an increasing number of terms in the expansion. It is clear that the series quickly becomes inaccurate with a larger number of terms included.

\section{Fourier expansions}

In order better to understand the poor convergence properties of the Gram-Charlier series, let us first discuss how it is related to the Fourier expansion. A Fourier expansion (Szegö 1978; Suetin 1979; Nikiforov \& Uvarov 1988) for any function $f(x)$ in the set of orthogonal polynomials $P_{n}$ is given by

$f(x) \sim \sum_{n=0}^{\infty} a_{n} P_{n}(x)$,

with

$a_{n}=\frac{1}{h_{n}} \int_{-\infty}^{\infty} w(t) f(t) P_{n}(t) \mathrm{d} t$.

Here $h_{n}$ is the squared norm

$h_{n}=\int_{-\infty}^{\infty} w(t) P_{n}^{2}(t) \mathrm{d} t$,
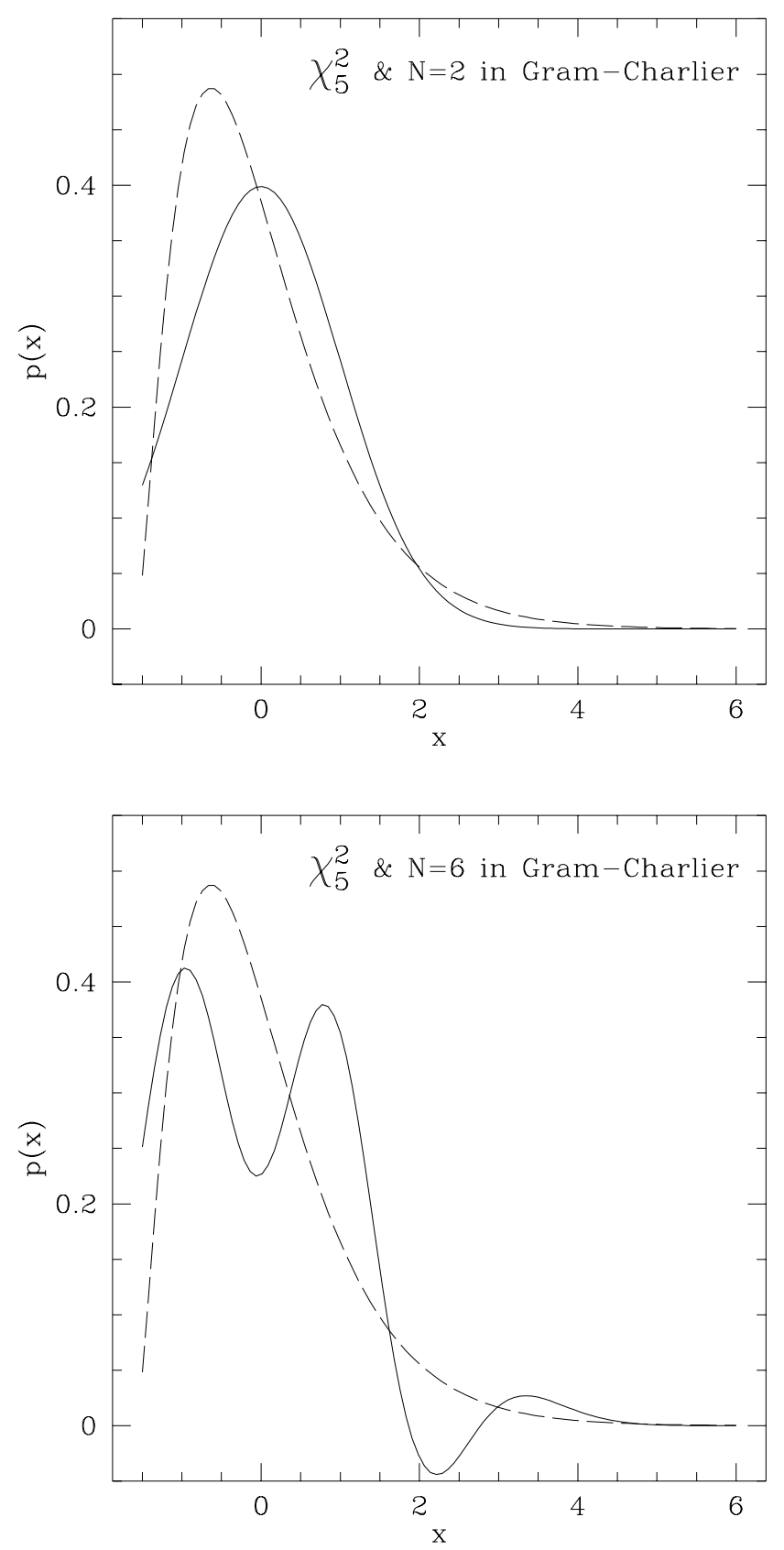

Fig. 1. The normalized $\chi^{2} \operatorname{PDF}$ (15) for $\nu=5$ (dashed line), and its Gram-Charlier approximations with 2 and 6 terms in the expansion (solid line)

and

$h_{n}=\sqrt{2 \pi} n ! \quad$ for $\quad H e_{n}(x)$,

$h_{n}=\sqrt{\pi} 2^{n} n$ ! for $\quad H_{n}(x)$.

Now we can see that the Gram-Charlier series (11) is just the Fourier expansion (16) of $f(x)=p(x) / Z(x)$ in the set of Chebyshev-Hermite polynomials with $c_{n}=(-1)^{n} a_{n}$.

The properties of the Gram-Charlier approximations of $p(x)$ in Figs. 1 and 2 are to be considered in the general context of the convergence of Fourier expansions. 

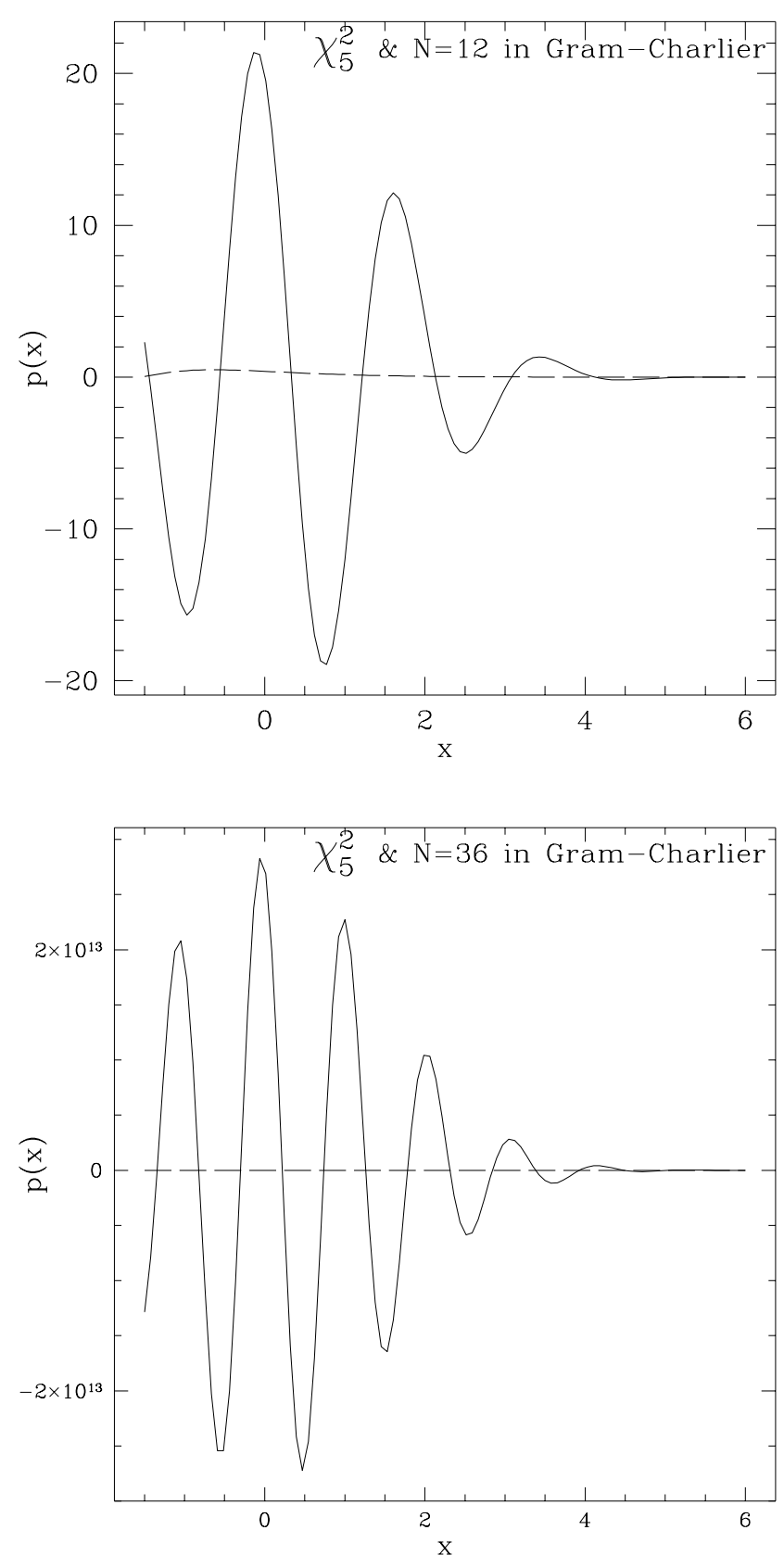

Fig. 2. The same as in Fig. 1 but for 12 and 36 terms in the Gram-Charlier expansion

The source of the divergence lies in the sensitivity of the Gram-Charlier series to the behavior of $p(x)$ at infinity - the latter must fall to zero faster than $\exp \left(-x^{2} / 4\right)$ for the series to converge (Cramér 1957; Kendall 1952). This is often too restrictive for practical applications. Our example of the $\chi^{2}$ distribution in (15), with its exponential behavior at infinity, clearly demonstrates this.

The Fourier expansion of $p(x) / Z(x)$ in another set of Hermite polynomials $H_{n}(x)$ (8) (not in Chebyshev-
Hermite polynomials $\mathrm{He}_{n}$ (7), as for the Gram-Charlier series) is sometimes used:

$p(x) \sim \sum_{n=0}^{\infty} a_{n} H_{n}(x) Z(x)$,

with

$a_{n}=\frac{\sqrt{\pi}}{2^{n-1} n !} \int_{-\infty}^{\infty} Z(t) p(t) H_{n}(t) \mathrm{d} t$.

This series is often called the Gauss-Hermite expansion (see e.g. its application to spectral lines of galaxies in van der Marel \& Franx 1993). Examples in Figs. 3 and 4 show its better convergence.

Van der Marel \& Franx (1993) use a theorem due to Myller-Lebedeff on the convergence of the Gauss-Hermite expansion: it converges when $x^{3} p(x) \rightarrow 0$ for any $p(x)$ with finite and continuous second derivative. Actually, the conditions sufficient for the convergence are better: if $p(t)$ obeys the Lipschitz condition

$|p(t)-p(x)| \leq M|t-x|^{\alpha}, \quad M=$ const,$\quad 0<\alpha \leq 1$,

in a vicinity of $x$ and

$\int_{-\infty}^{\infty}|p(t)|\left(1+|t|^{3 / 2}\right) \mathrm{d} t<\infty$

then the Gauss-Hermite series converges to $p(x)$ at $x$ (see e.g. Suetin 1979). These weaker conditions imply that the class of PDFs with convergent Gauss-Hermite expansions is much wider than suggested by the Myller-Lebedeff theorem cited in van der Marel \& Franx (1993). But the simple relation between the coefficients in the expansion and the moments of the PDF typical for the Gram-Charlier series is now lost (cf. Eqs. (12), (13) and (22)). It might be not important in many practical applications when the moments cannot be accurately determined from observations, but it is very important for a theoretical work based on the analysis of the moments.

Our Figs. 3 and 4 show that the $\chi^{2}$ PDF is well-suited for approximation by a Gauss-Hermite series. However, one should be cautious about the accuracy of the computation of the Fourier coefficients for higher order terms. We have not encountered the problem of numerical errors in our Gram-Charlier example, since there all coefficients can be calculated analytically. Yet in general care must be taken of the computational accuracy to avoid spurious numerical divergence of a series which converges theoretically.

\section{The Edgeworth asymptotic expansion}

A random variable $X$ can be normalized to unit variance by dividing by its standard deviation $\sigma$. The Edgeworth expansion is a true asymptotic expansion of the PDF of the normalized variable $X / \sigma$ in powers of the parameter $\sigma$, whereas the Gram-Charlier series is not. This difference between the Gram-Charlier series and the Edgeworth expansion was pointed out by Juszkiewicz et al. (1995), and 

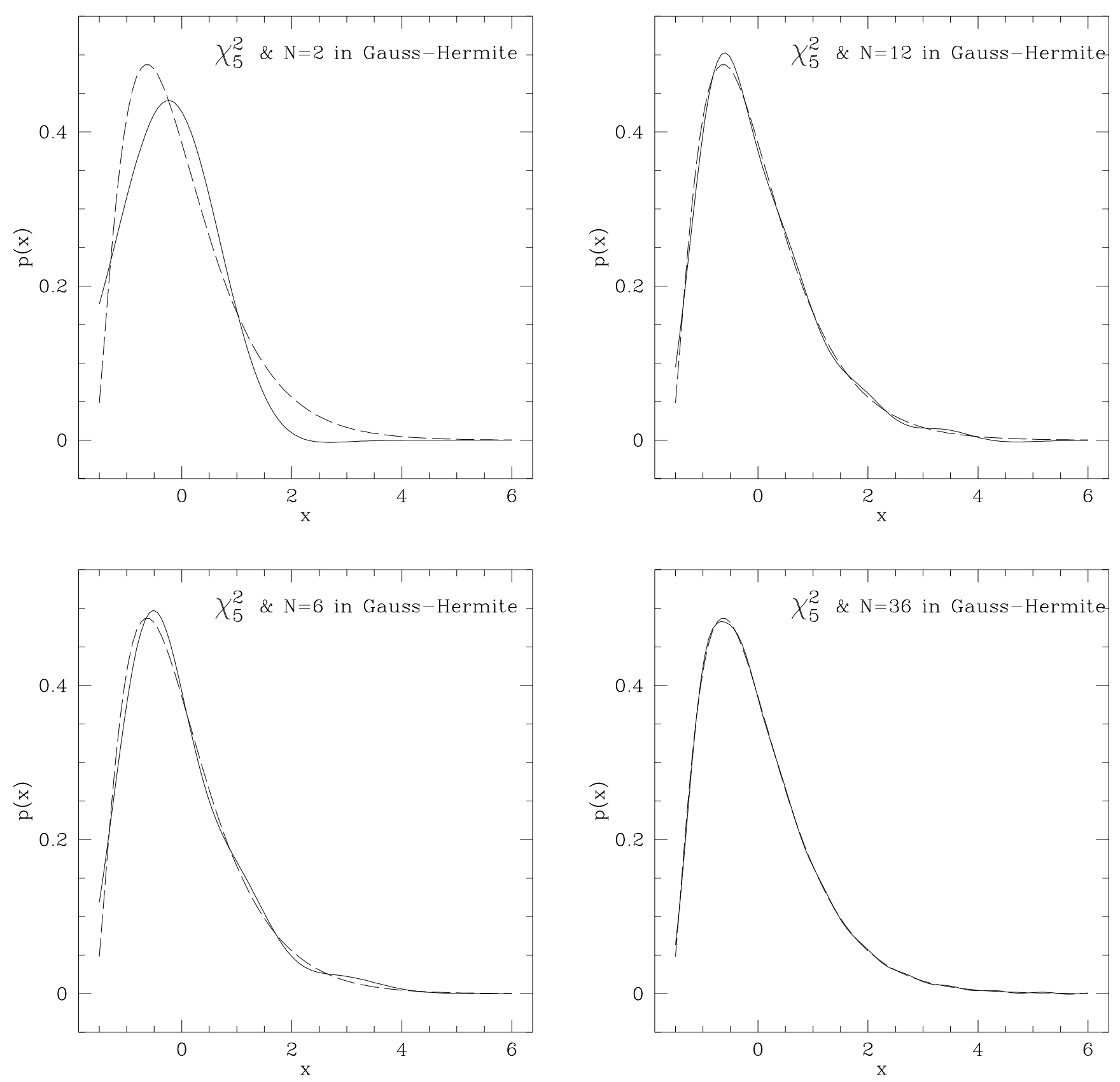

Fig. 3. The normalized $\chi^{2} \operatorname{PDF}$ (15) for $\nu=5$ (dashed line), and its Gauss-Hermite approximations with 2 and 6 terms in the expansion (solid line)

in independent work by Bernardeau \& Kofman (1995), followed by Amendola (1994) and Colombi (1994).

Juszkiewicz et al. (1995) and Bernardeau \& Kofman (1995) use 2 or 3 terms of the Edgeworth expansion derived e.g. in Cramér (1957). We note that the full explicit expansion for arbitrary order $s$ was obtained already in 1962 by Petrov (1962), see also Petrov $(1972,1987)$.

Petrov derived a powerful generalization of the Edgeworth expansion for a sum of random variables.

Fig. 4. The same as in Fig. 3 but for 12 and 36 terms in the Gauss-Hermite expansion

In this section we give a simplified derivation of the Edgeworth series, following Petrov (1972). This derivation, for an arbitrary order, is somewhat simpler than the derivation given for example by Bernardeau \& Kofman (1995) for the third order only of the Edgeworth expansion.

The characteristic function $\Phi(t)$ of a random variable $X$ is the expectation $\operatorname{Eexp}(i t X)$ as a function of $t$,

$\Phi(t) \equiv \int_{-\infty}^{\infty} \mathrm{e}^{i t x} \mathrm{~d} F(x)$ 

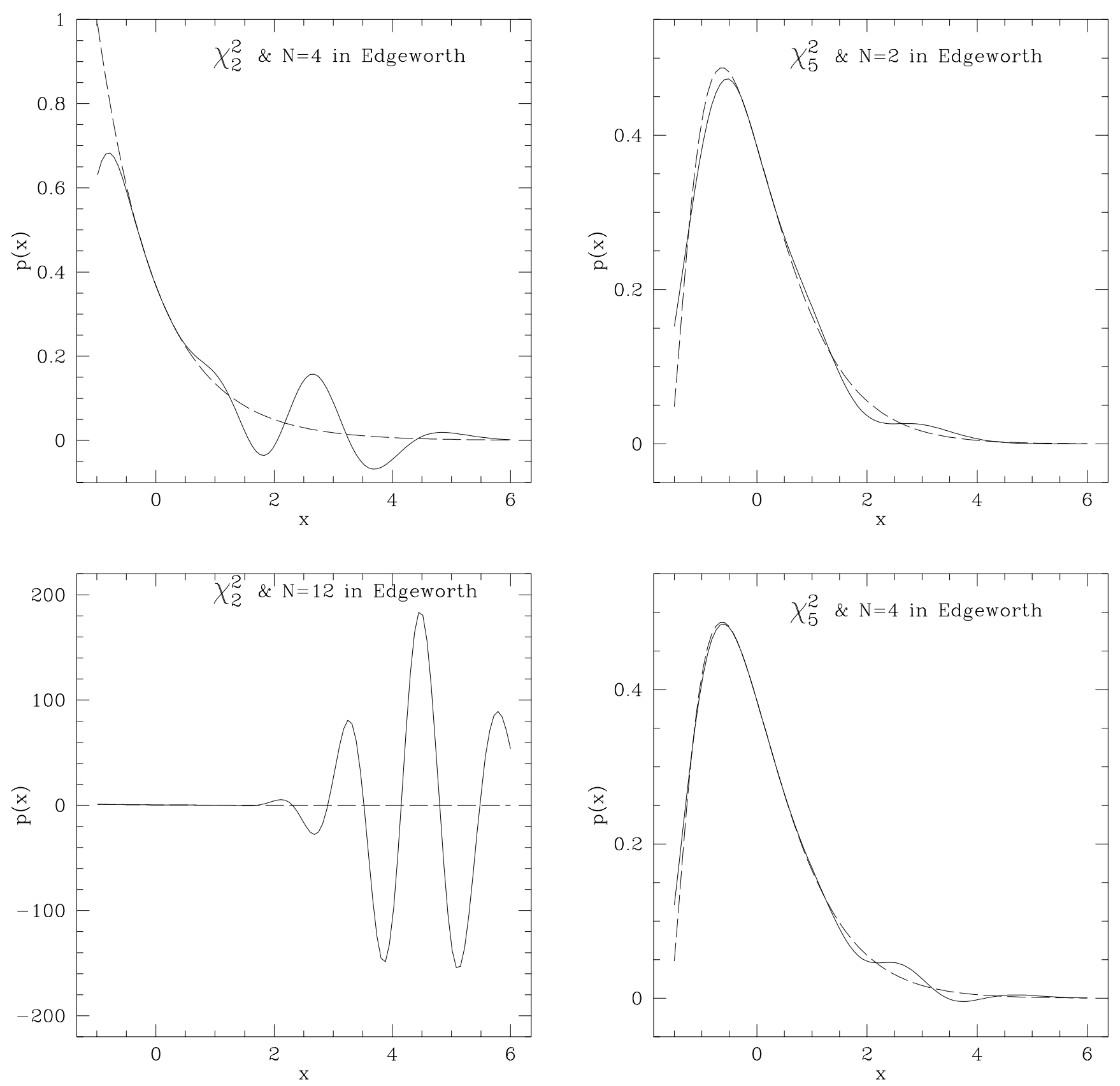

Fig. 5. The normalized $\chi^{2} \operatorname{PDF}(15)$ for $\nu=2$ (dashed line), and its Edgeworth-Petrov approximations with 4 and 12 terms in the expansion (solid line)

Fig. 6. The normalized $\chi^{2} \operatorname{PDF}(15)$ for $\nu=5$ (dashed line), and its Edgeworth-Petrov approximations with 2 and 4 terms in the expansion (solid)

that is the Fourier transform of $p(x)$ if the probability density $p(x)=\mathrm{d} F(x) / \mathrm{d} x$ exists. The definition (25) implies that if the moment $\alpha_{k}(2)$ of $X$ exists,

$\Phi^{(k)}(0)=i^{k} \alpha_{k}$.

Hence the Taylor series for $\Phi(t)$ is given by

A similar series for $\ln \Phi(t)$,

$\ln \Phi(t) \sim \sum_{n=1}^{\infty} \frac{\kappa_{n}}{n !}(i t)^{n}$,

involves cumulants (semi-invariants) $\kappa_{n}$ defined by

$\kappa_{n} \equiv \frac{1}{i^{n}}\left[\frac{\mathrm{d}^{n}}{\mathrm{~d} t^{n}} \ln \Phi(t)\right]_{t=0}$.

$\Phi(t) \sim 1+\sum_{k=1}^{\infty} \frac{\alpha_{k}}{k !}(i t)^{k}$.

In Appendix A: we prove a fundamental lemma of calculus for the $n$-th derivative of a composite function $f \circ g(x) \equiv$ 
$f(g(x))$, which reads

$$
\begin{aligned}
& \frac{\mathrm{d}^{n}}{\mathrm{~d} x^{n}} f(g(x))= \\
& \left.\quad n ! \sum_{\left\{k_{m}\right\}} f^{(r)}(y)\right|_{y=g(x)} \prod_{m=1}^{n} \frac{1}{k_{m} !}\left(\frac{1}{m !} g^{(m)}(x)\right)^{k_{m}}
\end{aligned}
$$

where $r=k_{1}+k_{2}+\ldots+k_{n}$ and the set $\left\{k_{m}\right\}$ consists of all non-negative integer solutions of the Diophantine equation

$k_{1}+2 k_{2}+\ldots+n k_{n}=n$.

Using (30) we derive a useful relation (Petrov 1987) between the cumulants $\kappa_{n}$ and the moments $\alpha_{k}$ of a PDF in Appendix B:,

$\kappa_{n}=n ! \sum_{\left\{k_{m}\right\}}(-1)^{r-1}(r-1) ! \prod_{m=1}^{n} \frac{1}{k_{m} !}\left(\frac{\alpha_{m}}{m !}\right)^{k_{m}}$.

Here summation extends over all non-negative integers $\left\{k_{m}\right\}$ satisfying $(31)$ and $r=k_{1}+k_{2}+\ldots+k_{n}$. We describe a simple algorithm for obtaining all solutions of Eq. (31) in Appendix C.

Now we are ready to begin with the derivation of the Edgeworth expansion. Consider a random variable $X$ with $\mathrm{E} X=0$ (this can always be achieved by an appropriate choice of origin), and let $X$ have dispersion $\sigma^{2}$. If $X$ has the characteristic function $\Phi(t)$, then the normalized random variable $X / \sigma$ has the characteristic function $\varphi(t)=\Phi(t / \sigma)$. Therefore we have from Eqs. (28) and (29) that

$\ln \varphi(t)=\ln \Phi(t / \sigma) \sim \sum_{n=2}^{\infty} \frac{\kappa_{n}}{\sigma^{n} n !}(i t)^{n}$

Here the sum starts at $n=2$ because $\mathrm{E} X=0$. Moreover, since $\kappa_{2}=\sigma^{2}$ (see Eq. (32)) we obtain

$\varphi(t) \sim \mathrm{e}^{-t^{2} / 2} \exp \left\{\sum_{n=3}^{\infty} \frac{S_{n} \sigma^{n-2}}{n !}(i t)^{n}\right\}$,

with

$S_{n} \equiv \kappa_{n} / \sigma^{2 n-2}$.

Let us write the exponential function in (34) as a formal series in powers of $\sigma$,

$\exp \left\{\sum_{r=1}^{\infty} \frac{S_{r+2} \sigma^{r}}{(r+2) !}(i t)^{r+2}\right\} \sim 1+\sum_{s=1}^{\infty} \mathcal{P}_{s}(i t) \sigma^{s}$,

where the coefficient of the power $s$ is a function $\mathcal{P}_{s}(i t)$. Now, using $g(x) \equiv \sum_{r=1}^{\infty}\left\{S_{r+2}(i t)^{r+2} x^{r} /(r+2) !\right\}$ and $f \equiv$ exp in (30), we find that

$$
\begin{aligned}
\mathcal{P}_{s}(i t) & \left.\equiv \frac{1}{s !} \frac{\mathrm{d}^{s}}{\mathrm{~d} x^{s}} f(g(x))\right|_{x=0} \\
= & \sum_{\left\{k_{m}\right\}} \prod_{m=1}^{s} \frac{1}{k_{m} !}\left(\frac{S_{m+2}(i t)^{m+2}}{(m+2) !}\right)^{k_{m}},
\end{aligned}
$$

where the summation extends again over all non-negative integers $\left\{k_{m}\right\}$ satisfying (31). Thus the function $\mathcal{P}_{s}$ is just a polynomial.
Suppose that the probability density $p(x)$ of a random variable $X$ exists. Then the $\mathrm{PDF}$ for $X / \sigma$ is $q(x) \equiv$ $\sigma p(\sigma x)$, and it is the inverse Fourier transform of the characteristic function $\varphi$ :

$q(x)=\frac{1}{2 \pi} \int_{-\infty}^{\infty} \mathrm{e}^{-i t x} \varphi(t) \mathrm{d} t$.

If $\Phi(t)$ is the Fourier transform of a function $p(x)$, then $(-i t)^{n} \Phi(t)$ is the transform of the $n$-th derivative of $p(x)$,

$\frac{\mathrm{d}^{n}}{\mathrm{~d} x^{n}} p(x)=\frac{1}{2 \pi} \int_{-\infty}^{\infty} \mathrm{e}^{-i t x}(-i t)^{n} \Phi(t) \mathrm{d} t$

The Fourier transform of the Gaussian distribution $Z(x)$ in (5) is $\exp \left(-t^{2} / 2\right)$, see e.g. Bateman \& Erdélyi (1954). Therefore each $(i t)^{n}$, multiplied by $\exp \left(-t^{2} / 2\right)$ in the expansion of $\varphi$ (see Eqs. (34) to (37)), generates (according to Eq. 38$)$ the $n$-th derivative of $Z(x)$,

$(-1)^{n} \frac{\mathrm{d}^{n}}{\mathrm{~d} x^{n}} Z(x)=\int_{-\infty}^{\infty} \mathrm{e}^{-i t x}(i t)^{n} \exp \left(-t^{2} / 2\right) \mathrm{d} t$,

in the corresponding expansion for $q(x)$,

$q(x)=Z(x)+\sum_{s=1}^{\infty} \sigma^{s}$
$\times\left\{\sum_{\left\{k_{m}\right\}} \prod_{m=1}^{s} \frac{1}{k_{m} !}\left(\frac{S_{m+2}(-1)^{m+2}}{(m+2) !} \frac{\mathrm{d}^{m+2}}{\mathrm{~d} x^{m+2}}\right)^{k_{m}} Z(x)\right\}$.

Here the set $\left\{k_{m}\right\}$ in the sum consists of all non-negative integer solutions of the equation

$k_{1}+2 k_{2}+\ldots+s k_{s}=s$.

Using (10) and $r=k_{1}+k_{2}+\ldots+k_{s}$ we can rewrite (41) in terms of the Chebyshev-Hermite polynomials:

$$
\begin{aligned}
& q(x)=\sigma p(\sigma x)=Z(x)\left\{1+\sum_{s=1}^{\infty} \sigma^{s}\right. \\
& \left.\times \sum_{\left\{k_{m}\right\}} H e_{s+2 r}(x) \prod_{m=1}^{s} \frac{1}{k_{m} !}\left(\frac{S_{m+2}}{(m+2) !}\right)^{k_{m}}\right\} .
\end{aligned}
$$

This is the Edgeworth expansion for arbitrary order $s$. See Petrov $(1972,1987)$ for a more general form of the expansion (for non-smooth cumulative distribution functions $F(x)$ and for a sum of random variables) and for the proof that the series (43) is asymptotic (see also the classical references Cramér 1957 and Feller 1966). This means that if the first $N$ terms are retained in the sum over $s$, then the difference between $q(x)$ and the partial sum is of a lower order than the $N$-th term in the sum (Erdélyi 1956; Evgrafov 1961). Convergence plays no role in the definition of the asymptotic series.

Strictly speaking, Petrov (1972) proves the asymptotic theorems for sums of $\nu$ independent random variables only when $\sigma \sim 1 / \nu^{1 / 2}$, and not for any $\sigma$, which we used in our derivation. But in all practical applications where nearly Gaussian PDFs occur (and in all applications that we consider in the present work), those PDFs basically are the 


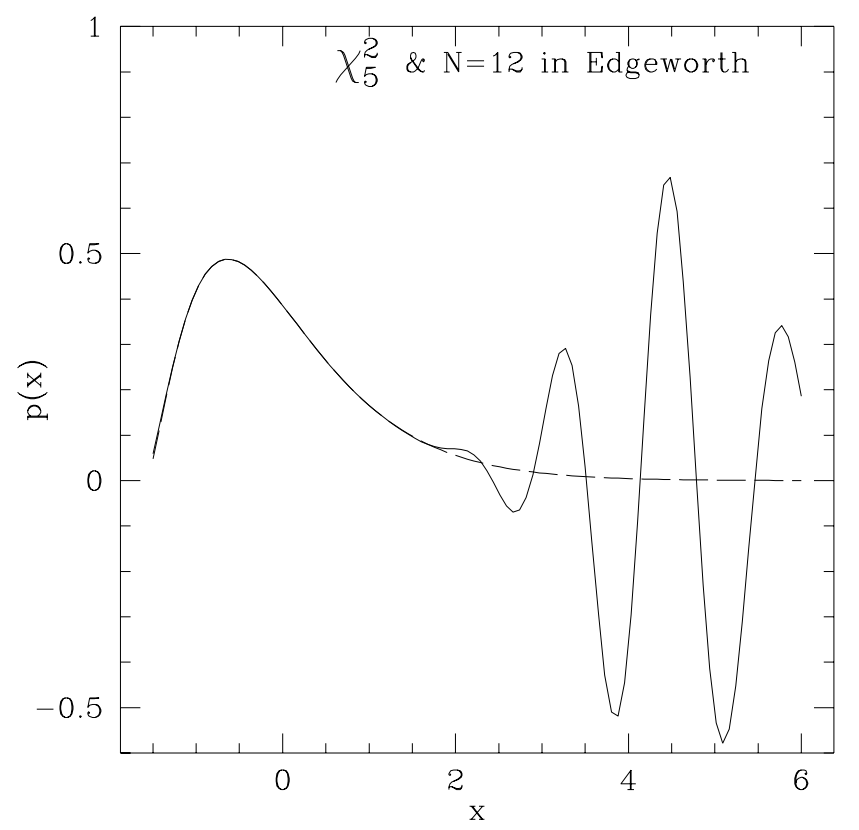

Fig. 7. The normalized $\chi^{2} \operatorname{PDF}$ (15) for $\nu=5$ (dashed line) and its Edgeworth-Petrov approximations with 12 terms in the expansion (solid)

sums of random variables, and the proofs of the asymptotic theorems are relevant. In the next section we show how the theory works in practice.

Figures 5-8 show some examples of the Edgeworth expansion for the $\chi^{2}$ distribution. It is clear that for strongly non-Gaussian cases, like $\chi_{\nu}^{2}$ for $\nu=2$, it has a very small domain of applicability in practical cases since it diverges like the Gram-Charlier series for a large number of terms (Fig. 5). But already in this case one can check that the order of the last term retained gives the order of the error correctly, and one can truncate the expansion when the last term becomes unacceptably large. For nearly Gaussian distributions the situation is much better: compare the cases for $\nu=5$ and $\nu=20$ in Figs. 6, 7 and 8 .

\section{Peculiar velocities from cosmic strings}

As an example we consider the probability distribution of peculiar velocities within the cosmic string model of structure formation. Cosmic strings are one-dimensional topological defects possibly formed in a phase transition in the early Universe (Brandenberger 1994; Hindmarsh \& Kibble 1995; Vilenkin \& Shellard 1994). After the time of formation, the string network quickly evolves to a scaling solution with a constant number $n_{s}$ of strings passing through a Hubble volume in one expansion time.

Since individual topological defects give rise to velocity perturbations of the dark matter through which they move
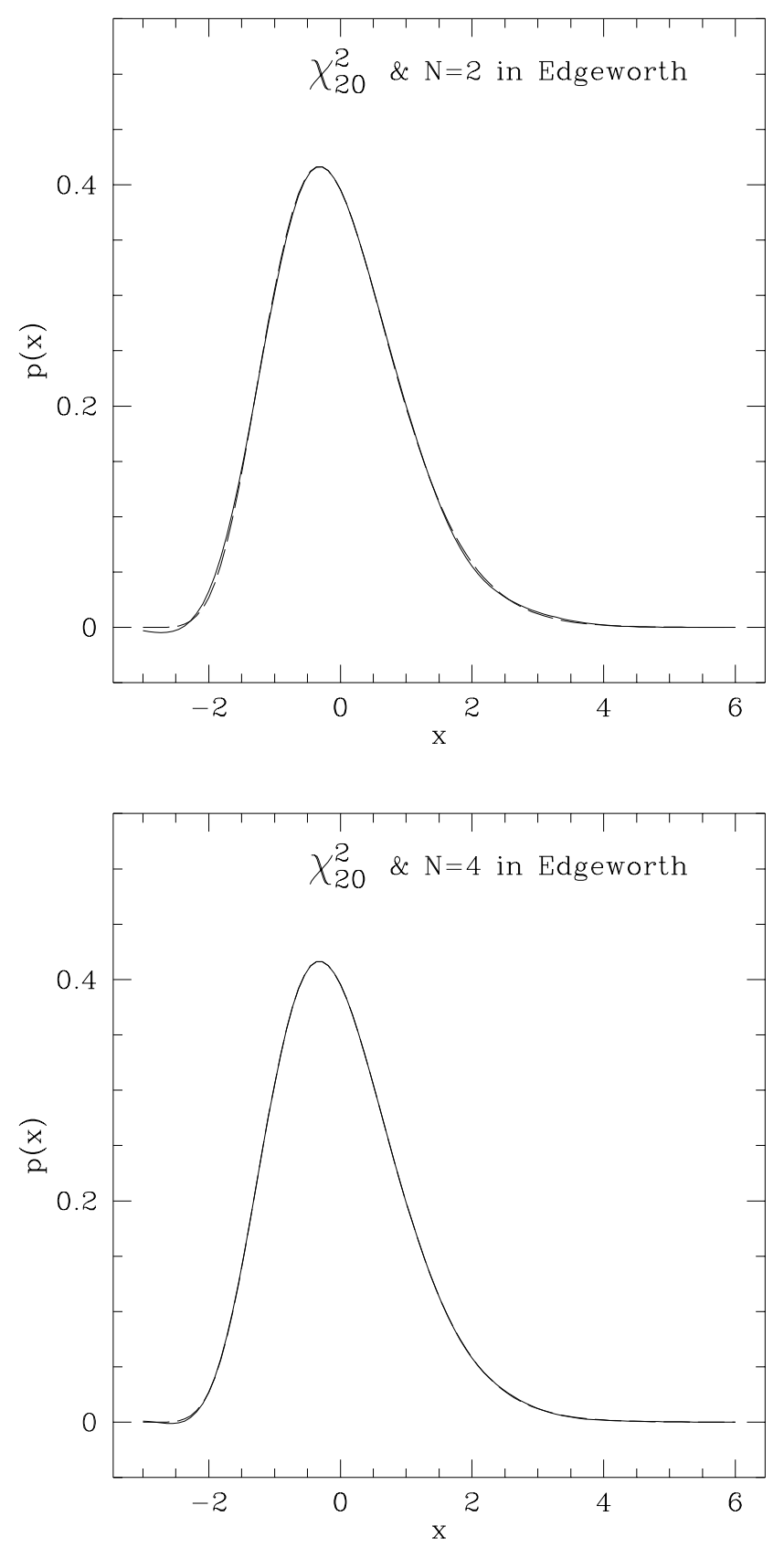

Fig. 8. The normalized $\chi^{2} \operatorname{PDF}(15)$ for $\nu=20$ (dashed line), and its Edgeworth-Petrov approximations with 2 and 4 terms in the expansion (solid)

up to a distance of a Hubble radius, one expects a nonGaussian result, in contrast to inflationary theories which predict a Gaussian PDF. Therefore departures from a normal distribution may be a way to distinguish between the two main classes of theories of structure formation, inflation and topological defects. However, since many strings present between the time of equal matter and radiation contribute to the perturbations, a nearly Gaussian PDF can result due to the central limit theorem. 


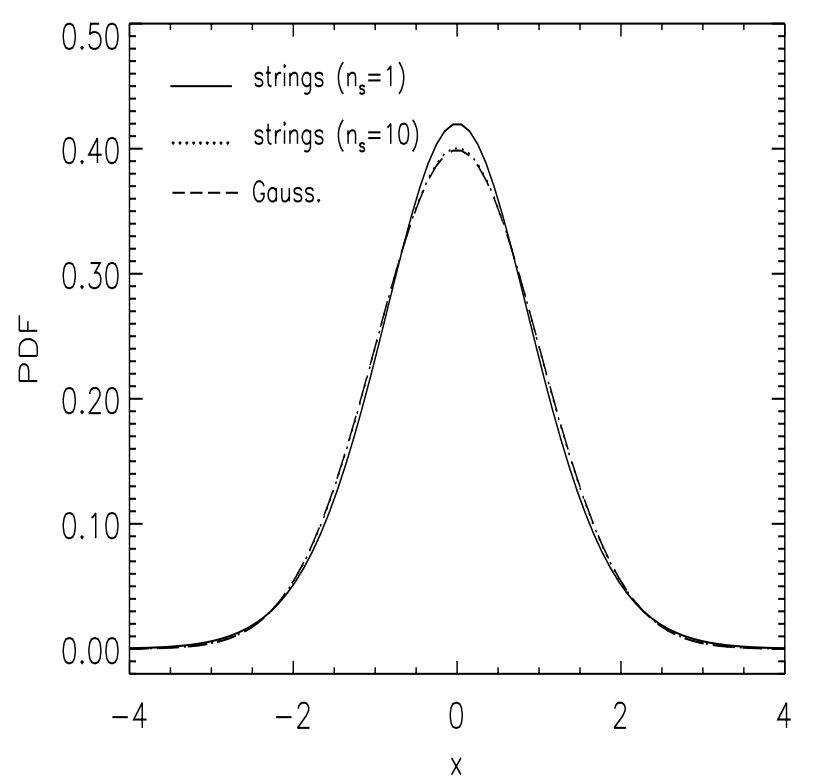

Fig. 9. Edgeworth expansion up to 10th order for the PDF of peculiar velocities from cosmic strings, within an analytical model for the string network, for two values of the number of strings per Hubble volume (Moessner 1995)

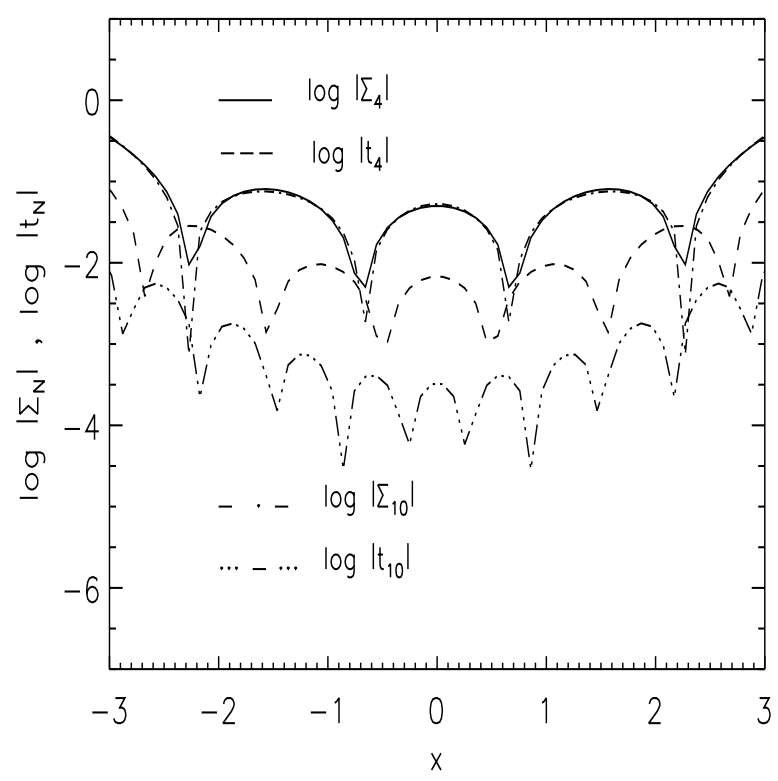

Fig. 10. Relative deviation $\Sigma_{N}$ from a normal distribution of the Edgeworth expansion up to $N$ th order of the PDF of peculiar velocities from cosmic strings, for $n_{s}=1$. Also shown is the error $t_{N}$ of this deviation associated with the expansion

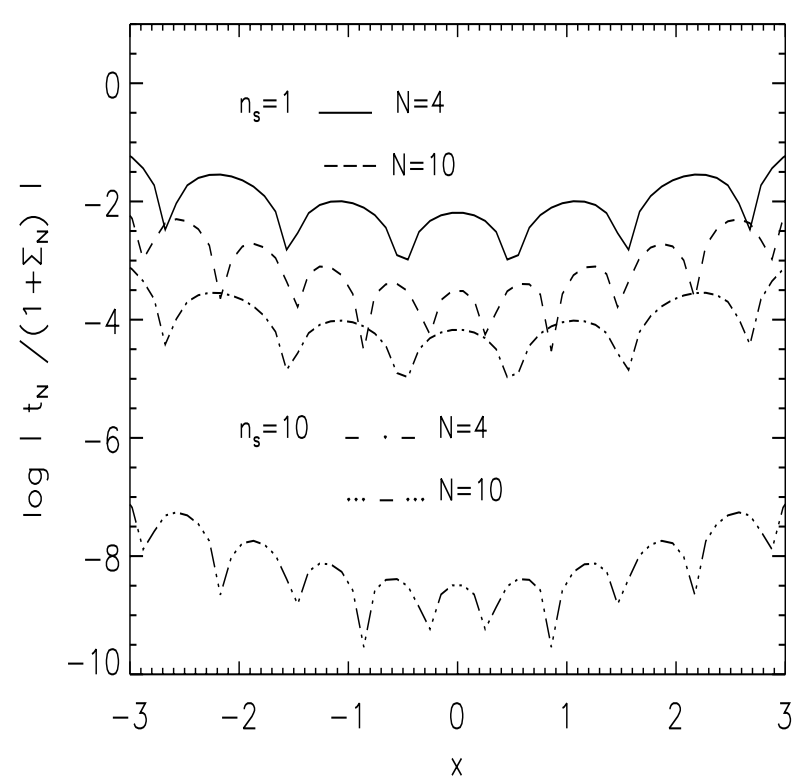

Fig. 11. Relative error $t_{N} /\left(1+\Sigma_{N}\right)$ in the Edgeworth expansion of $q(x) / Z(x)$ for the PDF of peculiar velocities from cosmic strings, for two values of $N$ and $n_{s}$

In order to estimate the deviation of the PDF from the normal distribution we use Petrov's formula (43) for the Edgeworth series in this section. We calculate the cumulants up to 12 th order in the analytical model for the cosmic string network presented in Moessner (1995), where the cumulants are given up to 8th order.

For simplicity, let us write Eq. (43) schematically as

$q(x)=Z(x)\left\{1+\sum_{s=1}^{\infty} t_{s}\right\}$

i.e. denote the $s$ th term in the sum by $t_{s}$. Let us further denote the sum up to $s=N$ by $\Sigma_{N} \equiv \sum_{s=1}^{N} t_{s}$. In Fig. 9 we show the Edgeworth expansion of the PDF of peculiar velocities up to 10th order, for the case of $n_{s}=1$ string per Hubble volume. Expanding up to $N$ th order, the relative deviation of the PDF from a normal distribution is given by,

$\frac{q(x)}{Z(x)}-1=\Sigma_{N}$.

It is only significant if the error of the asymptotic expansion, which is of the order of the last term included, is smaller than this deviation. In Fig. 10 we show the relative deviation $\Sigma_{N}$ and the error $t_{N}$ associated with it. The wiggles or cusps in the graphs appear at zeros of $\Sigma_{N}$ and $t_{N}$ which are both oscillating, changing their sign, and we have plotted the logarithms of the absolute values. So only the maxima of the curves give a true indication of the deviations and errors. For $N=10$ the error is clearly below the deviation and thus the latter is significant. For 
$N=4$, the error is still below the deviation for most values of $x$, but it is not as clear, especially since the error is not exactly equal to $t_{N}$ but of that order only. In Fig. 11 we show the relative error $t_{N} /\left(1+\Sigma_{N}\right)$ in the expansion of $q(x) / Z(x)$. It is smaller for an expansion to higher order for a given number of strings per Hubble volume. The error decreases more strongly with $N$ for larger $n_{s}$. The relative error is also smaller, at fixed $N$, for a larger $n_{s}$, in which case the PDF is closer to a normal distribution.

It is interesting to compare these results with the general theory. Petrov (1972) proves that under certain conditions (which are fulfilled in most physically important cases)

$q(x)=Z(x)\left\{1+\sum_{s=1}^{N} t_{s}\right\}+o\left(\sigma^{N}\right)$

uniformly for $-\infty<x<+\infty$, when $\sigma \sim 1 / \nu^{1 / 2}$ and the PDF $q(x)$ is for a sum of $\nu$ random variables. One should remember that each $t_{s}$ is of the order of $\sigma^{s}$ in (43). In our case $\nu$ is just $n_{s}$, so the error of the truncated Edgeworth series scales as $\sim 1 / n_{s}^{N / 2}$. From Fig. 11 we can see that the error for the case of $n_{s}=10$ strings is indeed $N / 2$ orders less than for the case of $n_{s}=1$ string, i.e. 2 orders for $N=4$ and 5 orders for $N=10$ terms in the expansion. This is an illustration of the theory developed by Petrov (1972, 1987).

\section{Conclusions}

We have shown that the Gram-Charlier series has a limited domain of applicability for nearly normal distributions because of its rather poor convergence properties. The Gauss-Hermite expansion can give good results in problems like fitting profiles of spectral lines of galaxies, supernovae, or ordinary stars. In advanced calculations of stellar atmospheres (e.g. Hauschildt et al. 1997; Hubeny \& Lanz 1995) the profiles of thousands or even millions of lines must be integrated for up to hundreds of Doppler widths, and the Gauss-Hermite expansion can perhaps be useful for saving information of the line profiles in an economical way. But since it has no intrinsic measure of accuracy, the number of terms needed in the expansion must be examined carefully for each individual problem.

For situations where the estimate of a deviation of a PDF from a Gaussian one is needed, the asymptotic Edgeworth expansion is indispensable, and for high order moments the form of this expansion found by Petrov is necessary. We found a workable algorithm for Petrov's formula of the Edgeworth expansion and applied it to several examples. The source codes used in this work are available on request from the authors.

Acknowledgements. We are grateful to Ya.M. Kazhdan for valuable advice on references, to N.N. Pavlyuk for assistance, and to the referee for useful comments. The work of $\mathrm{SB}$ is supported in part by INTAS grant "Thermonuclear
Supernovae" and by a grant from the Research Center for the Early Universe, University of Tokyo, and he cordially thanks K. Nomoto, as well as W.Hillebrandt, MPA, Garching, for their hospitality.

This research has made use of NASA's Astrophysics Data System Abstract Service.

\section{Appendices}

\section{Appendix A: Lemma}

In Sect. 5, the relation (30) for the $n$-th derivative of a composite function $f \circ g(x) \equiv f(g(x))$ is used for the derivation of the Edgeworth asymptotic expansion. Here a simplified derivation of Eq. (30) is given. Petrov (1972, 1987) suggests a proof by induction. We note that this derivation is more transparent if one simply considers the Taylor expansion for $f \circ g$ expressed in terms of the Taylor expansions of $f$ and $g$ - see Bourbaki (1958). We have

$f(y)=f\left(y_{0}\right)+\frac{f^{\prime}}{1 !} \Delta y+\frac{f^{\prime \prime}}{2 !} \Delta^{2} y+\cdots \frac{f^{(n)}}{n !} \Delta^{n} y+\cdots$ and

$g(x)=g\left(x_{0}\right)+\frac{g^{\prime}}{1 !} \Delta x+\frac{g^{\prime \prime}}{2 !} \Delta^{2} x+\cdots \frac{g^{(m)}}{m !} \Delta^{m} x+\cdots$

Truncating the expansions at some $n$ and $m$ we find that $f \circ g(x)=f\left(g\left(x_{0}\right)\right)$

$$
\begin{aligned}
& +\frac{f^{\prime}}{1 !}\left(\frac{g^{\prime}}{1 !} \Delta x+\frac{g^{\prime \prime}}{2 !} \Delta^{2} x+\cdots \frac{g^{(m)}}{m !} \Delta^{m} x+\cdots\right) \\
& +\cdots \\
& +\frac{f^{(n)}}{n !}\left(\frac{g^{\prime}}{1 !} \Delta x+\frac{g^{\prime \prime}}{2 !} \Delta^{2} x+\cdots \frac{g^{(m)}}{m !} \Delta^{m} x+\cdots\right)^{n} .
\end{aligned}
$$

On the other hand, we can write down the Taylor series for the composite function,

$f \circ g(x)=f \circ g\left(x_{0}\right)$

$$
+\frac{(f \circ g)^{\prime}}{1 !} \Delta x+\frac{(f \circ g)^{\prime \prime}}{2 !} \Delta^{2} x+\cdots \frac{(f \circ g)^{(s)}}{s !} \Delta^{s} x+\cdots
$$

Now using the polynomial theorem,

$\left(x_{1}+x_{2}+\cdots+x_{m}\right)^{r}$

$$
\begin{aligned}
& =\sum_{\left\{k_{m}\right\}} \frac{r !}{k_{1} ! k_{2} ! \cdots k_{m} !} x_{1}^{k_{1}} x_{2}^{k_{2}} \cdots x_{m}^{k_{m}} \\
& =\sum_{\left\{k_{m}\right\}} r ! \prod_{s=1}^{m} \frac{x_{s}^{k_{s}}}{k_{s} !}
\end{aligned}
$$

where summation extends over all sets of non-negative integers $\left\{k_{m}\right\}$ satisfying $r=k_{1}+k_{2}+\ldots+k_{s}$, and comparing the terms $\Delta^{s} x$ with equal $s$ in (A4) and (A4), we obtain $\frac{\mathrm{d}^{s}}{\mathrm{~d} x^{s}} f(g(x))=$

$\left.s ! \sum_{\left\{k_{m}\right\}} f^{(r)}(y)\right|_{y=g(x)} \prod_{m=1}^{s} \frac{1}{k_{m} !}\left(\frac{1}{m !} g^{(m)}(x)\right)^{k_{m}}$.

This is the relation (30) which we sought. Here the set $\left\{k_{m}\right\}$ consists of non-negative solutions of the Diophantine equation

$k_{1}+2 k_{2}+\ldots+s k_{s}=s$, 


\section{Appendix B: Applications of the Lemma}

If we apply lemma (30) to Chebyshev-Hermite polynomials in $(7)$, we get $g(x)=-x^{2} / 2$ for $f=\exp$, so that only the terms with $m=1$ and $m=2$ are non-zero in the product in (30), and we only need non-negative integers $\left\{k_{1}, k_{2}\right\}$ as the solutions for $k_{1}+2 k_{2}=n$. Thus for each $k \equiv k_{2}$ running from 0 to $[n / 2]$ (entier of $n / 2$ ) we have $k_{1}=n-2 k$ and $r=n-k$. Finally, we have from (30) that

$$
\begin{aligned}
\frac{\mathrm{d}^{n}}{\mathrm{~d} x^{n}} \exp ( & \left.-x^{2} / 2\right)=n ! \sum_{k=0}^{[n / 2]} \mathrm{e}^{-x^{2} / 2} \frac{1}{(n-2 k) !} \\
& \times\left(\frac{1}{1 !}(-x)\right)^{n-2 k} \frac{1}{k !}\left(\frac{1}{2 !}(-1)\right)^{k},
\end{aligned}
$$

and the explicit expression (13) follows immediately from Rodrigues' formula (7).

Among other consequences of (30) is the relation (32) between cumulants $\kappa_{n}$ and moments $\alpha_{k}$ of a PDF. From the definition (29) we obtain this relation by simply applying (30) to the case of $f \equiv \ln$ and $g \equiv \Phi$. Since $\left.f^{(r)}(y)\right|_{y=g(t)}=(-1)^{r-1}(r-1) ! /\left.\Phi^{r}\right|_{t=0}=(-1)^{r-1}(r-1) !$, we find that

$\kappa_{n}=\left.\frac{1}{i^{n}} \frac{\mathrm{d}^{n}}{\mathrm{~d} t^{n}} \ln \Phi\right|_{t=0}=$

$\frac{n !}{i^{n}} \sum_{\left\{k_{m}\right\}}(-1)^{r-1}(r-1) ! \prod_{m=1}^{n} \frac{1}{k_{m} !}\left(\left.\frac{1}{m !} \Phi^{(m)}\right|_{t=0}\right)^{k_{m}}$.

Thus, from (26),

$\kappa_{n}=\frac{n !}{i^{n}} \sum_{\left\{k_{m}\right\}}(-1)^{r-1}(r-1) ! \prod_{m=1}^{n} \frac{i^{m k_{m}}}{k_{m} !}\left(\frac{\alpha_{m}}{m !}\right)^{k_{m}}$,

which is equivalent to (32). Here the sum extends over all non-negative integers $\left\{k_{m}\right\}$ satisfying (31) and $r=$ $k_{1}+k_{2}+\ldots+k_{n}$.

\section{Appendix C: Algorithm for computing indices $\boldsymbol{k}_{m}$}

To find all the solutions of Eq. (31) it is desirable to order all sets of non-negative integers $\left\{k_{m}\right\}$ satisfying it. We first rewrite (31) as

$n k_{n}+\ldots+2 k_{2}+k_{1}=n$.

It is natural to establish the ordering of the sets $S_{i} \equiv\left\{k_{m}\right\}$ satisfying Eq. (31) according to the order of numbers in their decimal representation. For $n=3$, say, we have 3 non-negative solutions

$$
\begin{aligned}
& S_{1}=\left\{k_{3}=0, k_{2}=0, k_{1}=3\right\} \\
& S_{2}=\left\{k_{3}=0, k_{2}=1, k_{1}=1\right\} \\
& S_{3}=\left\{k_{3}=1, k_{2}=0, k_{1}=0\right\}
\end{aligned}
$$

which can simply be written as

$$
S_{1}=003, S_{2}=011, S_{3}=100,
$$

and we say that $S_{1}<S_{2}<S_{3}$ since $3<11<100$. For $n \geq 10$, when the base 10 is no longer convenient, the sets of solutions can be ordered according to the order of the integer numbers

$k_{n} r^{n-1}+\ldots+k_{2} r+k_{1}$

for any natural base $r$. Those numbers are not important in themselves, what matters is thinking about the sets of $\left\{k_{m}\right\}$ as numbers in an abstract representation to base $r$.

On entry to our algorithm we have an integer $\mathrm{n}>$ 0 , and on exit we wish to have the number nsol of all solutions of Eq. (31) and the solutions themselves. For a set $S_{i}$ we introduce an abstract variable $\mathrm{S}$ to describe the array $k(1: n)$ of $n$ integer elements ordered as "quasidecimal digits".

So, in an abstract form the algorithm looks like:

$$
\begin{aligned}
& \text {-- } \quad \text { Statement QS : } \\
& \text {-- } \quad \text { all } \mathrm{S}<=\mathrm{S} \text { (CURRENT) } \\
& \text {-- } \quad \text { are out if and only if } \\
& --\quad \mathrm{k}(1)+2 * \mathrm{k}(2)+\ldots+\mathrm{n} * \mathrm{k}(\mathrm{n})=\mathrm{n} \\
& \text { <*initiate: make QS true for } \mathrm{S}=\mathrm{S} \text { (INITIAL) *>; } \\
& \text { _while <*condition: } \mathrm{S}{ }^{*}=\mathrm{S}\left(\text { FINAL) } *>_{\text {_do }}\right. \\
& <* \text { nextset: find next } \mathrm{S} \\
& \text { keeping QS invariant *> } \\
& \text { _od; -- Here } \mathrm{S}=\mathrm{S} \text { (FINAL) and } \mathrm{QS}=\text {. TRUE., i.e. } \\
& \text {-- all needed solutions are found }
\end{aligned}
$$

It is easy to initiate QS:

$$
\begin{aligned}
& \mathrm{k}(1)=\mathrm{n} ; \\
& \operatorname{mold}=1 ;-- \text { keeps largest } \mathrm{m} \\
& \quad-- \text { for non-zero } \mathrm{k}(\mathrm{m}) \\
& \text {-do } \mathrm{m}=2, \mathrm{n} ; \\
& \quad \mathrm{k}(\mathrm{m})=0 \\
& \text {-od; } \\
& \text { nsol=1; }
\end{aligned}
$$

The integer mold keeps track of the progress of the algorithm and allows us to establish the condition for the continuation of the main loop,

$$
\operatorname{mold} \neq \mathrm{n} \text {. }
$$

Finally, the core of the algorithm is the node nextset where we work with our sets as with digits, which is like doing the addition of decimal numbers by hand, column by column from right to left.

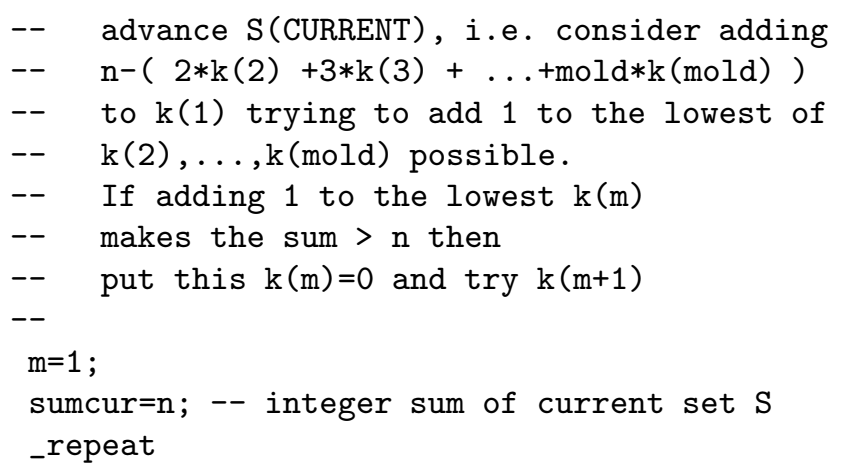


Table C1. Table of solutions to Eq. (31) for $n=1$ to 8. Zeros for higher orders are left blank

\begin{tabular}{rrrrr}
\hline $\mathrm{n}$ & 1 & 2 & 3 & 4 \\
\hline & 1 & 2 & 3 & 4 \\
& & 10 & 11 & 12 \\
& & 100 & 20 \\
& & & 101 \\
& & & & 1000 \\
\hline $\mathrm{n}$ & 5 & 6 & 7 & 8 \\
\hline & 5 & 6 & 7 & 8 \\
& 13 & 14 & 15 & 16 \\
21 & 22 & 23 & 24 \\
102 & 30 & 31 & 32 \\
110 & 103 & 104 & 40 \\
1001 & 111 & 112 & 105 \\
10000 & 200 & 120 & 113 \\
& 1002 & 201 & 121 \\
& 1010 & 1003 & 202 \\
& 10001 & 1011 & 210 \\
& 100000 & 1100 & 1004 \\
& & 10002 & 1012 \\
& & 10010 & 1020 \\
& & 100001 & 1101 \\
& & 1000000 & 2000 \\
& & & 10003 \\
& & & 10011 \\
& & & 10100 \\
& & & 100002 \\
& & & & 1000000010 \\
\hline & & &
\end{tabular}

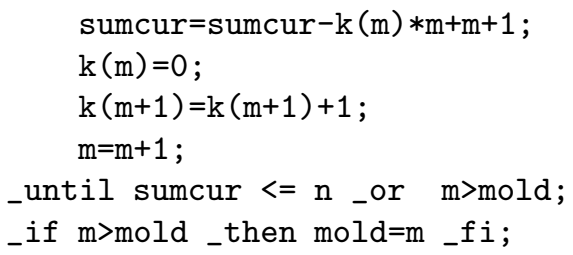

Here the node nextset ends, and the whole algorithm is finished. We have written it here in a pseudocode which can be translated mechanically into any machine language. We actually use a special preprocessor Trefor which automatically transforms the text above to standard Fortran (see Weinstein \& Blinnikov 1984, and Bartunov et al. 1997).

For reference, we present the first 8 sets of solutions found by this algorithm. In practice it is easier not to use the Table $\mathrm{C} 1$ even for low $\mathrm{n}$, but generate all coefficients in the code.

\section{References}

Abramowitz M., Stegun I., 1972, Handbook of Mathematical Functions. New York: Dover
Amendola L., 1994, ApJ 430, L9

Bartunov O.S., Blinnikov S.I., Popolitov V.A., 1997, Trefor: user's guide and references. Sternberg Astron. Inst. report

Bateman H., Erdélyi A., 1954, Tables of Integral Transforms. McGraw-Hill, New York, Toronto, London

Bernardeau F., 1992, ApJ 392, 1

Bernardeau F., 1994, A\&A 291, 697

Bernardeau F., Kofman L., 1995, ApJ 443, 479

Blinnikov S.I., 1996, Pis'ma v AZh 22, 92; translation: Astron. Lett. 22, 79

Bourbaki N., 1958, Éléments de mathématique. Livre IV. Fonctions d'une variable réelle (theórie élémentaire), Hermann \& Co., Paris, Ch. I, Sect. 3.2

Brandenberger R., 1994, Int. J. Mod. Phys. A9, 2117

Colombi S., 1994, ApJ 435, 536

Cramér H., 1957, Mathematical Methods of Statistics. Princeton Univ. Press, Princeton

Encyclopaedia of Mathematics, 1988, Translated from the Russian. Kluwer Academic Publishers, Dordrecht, Holland

Erdélyi A., 1956, Asymptotic Expansions, Dover, U.S.A.

Evgrafov M.A., 1961, Asymptotic estimates and entire functions, Translated from the Russian. Gordon and Breach, New York

Feller W., 1966, An Introduction to Probability Theory and its Applications, Vol. II, Ch. XVI. Wiley \& Sons, New York, London, Sydney, Toronto

Ferreira P.G., Magueijo J., Silk J., 1997, Phys. Rev. D56, 4592

Gaztañaga E., Fosalba P., Elizalde E., 1997, Non-Gaussian traces in the variance from small scale CMB observations, astro-ph/9705116

Gerhard O.E., 1993, MNRAS 265, 213

Hauschildt P.H., Baron E., Allard F., 1997, ApJ 483, 390

Heyl J.S., Hernquist L., Spergel D.N., 1994, ApJ 427, 165

Hindmarsh M., Kibble T., 1995, Rep. Prog. Phys. 58, 477

Hubeny I., Lanz T., 1995, ApJ 439, 875

Juszkiewicz R., Weinberg D.H., Amsterdamski P., Chodorowski M., Bouchet F., 1995, ApJ 442, 39

Kendall M. C., 1952, The advanced theory of statistics, Vol. 1, Distribution theory. Fifth Edition Charles Griffin \& Co., London

Kofman L., Bertschinger E., Gelb J.M., Nussser A., Dekel A., 1994, ApJ 420, 44

Luo X., 1995, ApJ 439, 517

Matsubara T., Yokoyama J., 1996, ApJ 463, 409

Moessner R., 1995, MNRAS 277, 927

Moessner R., Perivolaropoulos L., Brandenberger R., 1994, ApJ 425, 365

Nikiforov A.F., Uvarov V.B., 1988, Special Functions of Mathematical Physics, translated from the Russian. Birkhauser, Basel, Boston

Petrov V.V., 1962, Vestnik Leningrad. Univ. No. 19, 150-153

Petrov V.V., 1972, Summy nezavisimykh sluchainykh velichin, Moskva, Nauka; translation: Petrov V.V. Sums of independent random variables. Series title: Ergebnisse der Mathematik und ihrer Grenzgebiete; Vol. 82., 1975. Springer-Verlag, Berlin, New York

Petrov V.V., 1987, Predel'nye teoremy dlia summ nezavisimykh sluchainykh velichin. Moskva, Nauka; translation: Petrov V.V., Limit theorems of probability theory: sequences of independent random variables. Series title: Oxford studies in probability; 4, 1995. Clarendon Press, Oxford, Oxford University Press, New York 
Rix H., White S.D.M., 1992, MNRAS 254, 389

Scherrer R., Bertschinger E., 1991, ApJ, 381, 349

Suetin P.K., 1979, Klassicheskie Ortogonal'nye Mnogochleny, Moscow: Nauka

Szegö G., 1978, Orthogonal Polynomials. Providence: American Mathematical Society colloquium publications XXIII
Tabeling P., Zocchi G., Belin F., Maurer J., Willaime H., 1996, Phys. Rev. E. 53, 1613

van der Marel R.P., Franx M., 1993, ApJ 407, 525

Vilenkin A., Shellard E.P.S., 1994, Cosmic strings and other topological defects. Cambridge Univ. Press, Cambridge

Weinstein S.I., Blinnikov S.I., 1984, Trefor - a preprocessor for structured program design in Fortran. Report ITEP 198479 